\title{
Pulmonary arterial enlargement predicts cardiopulmonary complications after pulmonary resection for lung cancer: a retrospective cohort study
}

Keisuke Asakura ${ }^{1 *}$, Shota Mitsuboshi ${ }^{2}$, Makoto Tsuji ${ }^{2}$, Hiroyuki Sakamaki ${ }^{1}$, Sotaro Otake ${ }^{1}$, Shinsaku Matsuda ${ }^{1}$, Kaoru Kaseda ${ }^{2}$ and Kenichi Watanabe ${ }^{2}$

\begin{abstract}
Background: The finding of pulmonary arterial enlargement on computed tomography has been reported to be associated with pulmonary hypertension. On the other hand, pulmonary hypertension is a known risk factor for thoracic surgery. We investigated whether pulmonary arterial enlargement predicts cardiopulmonary complications following pulmonary resection for lung cancer.

Methods: We reviewed 237 consecutive patients who underwent pulmonary resection for lung cancer. Preoperative patient characteristics (sex, age, Brinkman index, cardiopulmonary comorbidities, cardiothoracic ratio, pulmonary function, and pulmonary arterial enlargement) and surgical data (surgical procedure, pathological stage, postoperative complications, mortality, and length of postoperative hospital stay) were analyzed. In order to evaluate preoperative pulmonary arterial enlargement, we measured the diameter of the main pulmonary artery at its bifurcation and that of the ascending aorta at its widest point using chest computed tomography and calculated the ratio of the former diameter to the latter.

Results: In all, 16 patients developed postoperative cardiopulmonary complications and 221 did not. One patient died from postoperative pneumonia. The mean age of patients who developed postoperative cardiopulmonary complications was significantly higher than that of those who did not ( $78 \pm 5$ years vs $69 \pm 9$ years, $P=0.0001$ ). The pulmonary artery-to-ascending-aorta ratio was significantly higher in patients who developed postoperative complications than in those who did not $(0.94 \pm 0.15$ vs. $0.81 \pm 0.11, P=0.03)$. Other preoperative patient characteristics and surgical data did not differ significantly between the groups. On multivariate analysis, pulmonary artery-to-ascending-aorta ratio (0.1-point increase; odds ratio $2.3,95 \%$ confidence interval $1.5-3.5 ; P=0.0002)$ and age (1-year increase; odds ratio 1.2, $95 \%$ confidence interval $1.1-1.3 ; P=0.03$ ) were found to be independent predictors of postoperative cardiopulmonary complications.
\end{abstract}

Conclusions: A finding of pulmonary arterial enlargement on computed tomography is a potential predictor of postoperative cardiopulmonary complications after lung cancer surgery.

Keywords: Lung cancer, Pulmonary resection, Pulmonary artery, Computed tomography, Pulmonary arterial hypertension

\footnotetext{
* Correspondence: asakurakeisuke@gmail.com

'Division of Thoracic Surgery, Department of Surgery, School of Medicine,

Keio University, 35 Shinanomachi, Shinjuku-ku, Tokyo, Japan

Full list of author information is available at the end of the article
} 


\section{Background}

Surgery is the standard therapeutic option in patients with early-stage lung cancer. Despite advances in surgical techniques and perioperative care, postoperative complication rates remain as high as $23-41 \%$ [1-5]. To improve the safety of surgical treatment for lung cancer, it is important to identify which candidates are at high risk of postoperative complications.

Pulmonary hypertension has been shown to be a predictor of postoperative cardiopulmonary complication (PCC) and mortality after surgery [6-8]. Standard methods for measuring the pulmonary arterial pressure include Doppler echocardiography and cardiac catheterization. However, performing these examinations is not practical in all surgical candidates for reasons of invasiveness and cost. It has been reported that the results of computed tomographic examination for pulmonary arterial enlargement correlate with those of invasive or echocardiographic measurements of pulmonary arterial pressure [9-11]. In the present study, the relationship between a finding of pulmonary arterial enlargement on computed tomography and PCCs following pulmonary resection was retrospectively investigated to clarify the value of pulmonary arterial enlargement as a predictor of PCCs in lung cancer patients.

\section{Methods}

Between October 2008 and May 2013, 241 consecutive patients with primary lung cancer underwent pulmonary resection at Sagamihara Kyodo Hospital, a 400-bed acute-care hospital. For this study, records of 237 of these patients were reviewed retrospectively, with four patients who underwent pneumonectomy being excluded. The Sagamihara Kyodo Hospital Ethical Committee approved the study. In accordance with the Ethical Guidelines for Clinical Studies published by the Japanese Ministry of Health, Labour and Welfare, the Ethical Committee waived the need for individual patients to provide consent because of the retrospective nature of the study, because consent could not be obtained from all of the patients, and because individual patients were not identified in the study.

Preoperatively, all patients underwent computed tomography of the body, magnetic resonance imaging of the brain, ${ }^{18}$ F-fluoro-2-deoxyglucose positron emission tomography, electrocardiography, and spirometric pulmonary function tests. The spirometric pulmonary function tests included tests of forced expiratory volume in $1 \mathrm{~s}\left(\mathrm{FEV}_{1}\right)$ and diffusing capacity of the lung for carbon monoxide (DLCO). Postoperative $\mathrm{FEV}_{1}$ and DLCO (ppoFEV 1 and ppoDLCO) were calculated based on the number of segments to be removed. Data collected preoperatively included the patients' sex, age, Brinkman index, and cardiopulmonary comorbidities. At our institution, the eligibility criteria for pulmonary resection are a predicted ppoFEV $_{1}$ and ppoDLCO $>40 \%$ of the average values in normal individuals (i.e. $\% \mathrm{ppoFEV}_{1}$ and $\%$ ppoDLCO >40). Standard pulmonary resection was performed using video-assisted thoracic surgery with access via two skin incisions: an 8-cm incision used for manipulation and a $1.5-\mathrm{cm}$ incision in which the utility port was placed to allow access for the thoracoscope, staplers, and other instruments.

\section{Examination for pulmonary arterial enlargement}

Axial computed tomography was used to examine the pulmonary artery for enlargement. Measurements were performed by a physician (KA, MT, or SM) who did not know the patient's postoperative course. In accordance with previous publications $[12,11,9,10]$, we measured the diameter of the main pulmonary artery at the level of its bifurcation and that of the ascending aorta at its widest point, using the same image for both. The measuring point of the main pulmonary artery is at the rises of bilateral pulmonary arteries. The rises of the main pulmonary arteries are obvious on axial computed tomography in most patients, because the angles of outline of pulmonary arteries change sharply at the points (Fig. 1). Then, we calculated the ratio between the diameters (pulmonary artery-to-ascending-aorta ratio: PA/A ratio) as the index of pulmonary arterial enlargement. We also calculated PA/A ratios on computed tomography 3 months after surgery to assess postoperative changes.

\section{Postoperative cardiopulmonary complications}

PCCs of lung resection may include one or more of the following: (1) pneumonia, defined as the appearance of pulmonary infiltrate on radiography, combined with fever and leukocytosis; (2) acute respiratory distress syndrome; (3) respiratory insufficiency requiring tracheostomy; (4) respiratory failure requiring mechanical ventilation; (5) atelectasis requiring bronchoscopic intervention; (6) hypoxaemia requiring home oxygen therapy; (7) arrhythmias requiring medication; (8) coronary artery diseases (angina pectoris and myocardial infarction); (9) acute heart failure requiring medication $[13,14]$. Complications related to surgical technique, such as prolonged air leaks, bronchopleural fistulas and chylothorax, were not examined in this study.

\section{Statistical analyses}

Patients were divided into those who developed PCCs and those who developed none, and differences in patient characteristics between the two groups were tested using Fisher's exact test or the Mann-Whitney $U$-test. The cut-off PA/A ratio most likely to predict the occurrence of PCCs was determined by the receiver operating 


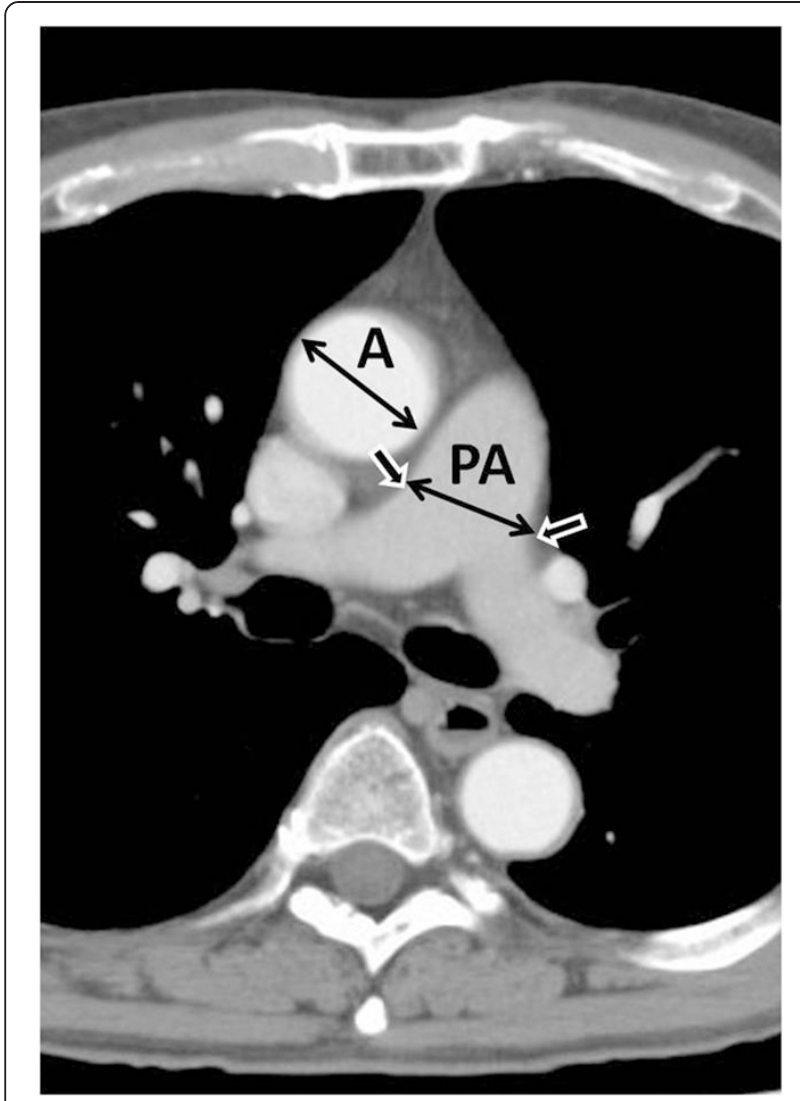

Fig. 1 Measurement of the diameters of the pulmonary artery and aorta. Axial chest computed tomographic image at the level of the bifurcation of the main pulmonary artery. Measurements of the diameter of the main pulmonary artery (PA) and that of the aorta (A) at the level of the bifurcation were used to calculate the PA/A ratio. Short arrows show the rises of the bilateral pulmonary arteries. It is the measuring point of the diameter of PA

characteristic (ROC) curve. Among patients who developed PCCs, the incidence of each PCC was compared between those with PA/A ratios above the cut-off value and those with PA/A ratios under it, and differences in PCCs between the two groups were tested using Fisher's exact test or the Mann-Whitney $U$ test. Factors appearing significant on these univariate analyses were further analyzed with multivariate logistic regression to determine if any were independent predictors of the occurrence of PCCs. All tests were two-sided and used a $5 \%$ significance level. All data were analyzed using SPSS software (SPSS Statistics 20, IBM, Armonk, NY, USA).

\section{Results}

Patient characteristics, surgical data, and postoperative cardiopulmonary complications

Preoperative patient characteristics and surgical data are summarized in Table 1. Of 237 patients who underwent pulmonary resection for lung cancer, 16 patients
Table 1 Preoperative patient characteristics and surgical data

\begin{tabular}{|c|c|c|c|c|}
\hline Characteristics & $\begin{array}{l}\text { Total } \\
(n=237)\end{array}$ & $\begin{array}{l}\text { No PCCs } \\
(n=221)\end{array}$ & $\begin{array}{l}\text { PCCs } \\
(n=16)\end{array}$ & $P$ value \\
\hline Age, years & $70 \pm 9$ & $69 \pm 9$ & $78 \pm 5$ & 0.0001 \\
\hline Male, n (\%) & $160(68)$ & $148(67)$ & $12(75)$ & 0.6 \\
\hline $\begin{array}{l}\text { Cardiopulmonary } \\
\text { comorbidity, n (\%) }\end{array}$ & $138(58)$ & $125(57)$ & $13(81)$ & 0.07 \\
\hline Cardiothoracic ratio & $0.47 \pm 0.05$ & $0.47 \pm 0.05$ & $0.48 \pm 0.08$ & 0.7 \\
\hline Brinkman index & $632 \pm 627$ & $620 \pm 603$ & $801 \pm 903$ & 0.6 \\
\hline$\% \mathrm{FEV}_{1}$ & $108 \pm 24$ & $109 \pm 23$ & $100 \pm 33$ & 0.2 \\
\hline $\mathrm{FEV}_{1} / \mathrm{FVC}$ & $71 \pm 11$ & $71 \pm 10$ & $68 \pm 17$ & 0.7 \\
\hline$\% D L C O$ & $105 \pm 31$ & $105 \pm 31$ & $103 \pm 31$ & 0.8 \\
\hline$\%$ ppoFEV 1 & $88 \pm 21$ & $87 \pm 21$ & $84 \pm 29$ & 0.4 \\
\hline \%ppoDLCO & $85 \pm 26$ & $85 \pm 26$ & $86 \pm 27$ & 0.9 \\
\hline PA/A ratio & $0.82 \pm 0.12$ & $0.81 \pm 0.11$ & $0.90 \pm 0.15$ & 0.03 \\
\hline Surgical procedure, n (\%) & & & & 1 \\
\hline $\begin{array}{l}\text { Lobectomy or } \\
\text { bilobectomy }\end{array}$ & $185(78)$ & $172(78)$ & $13(81)$ & \\
\hline $\begin{array}{l}\text { Segmentectomy or } \\
\text { wedge resection }\end{array}$ & $52(22)$ & $49(22)$ & $3(19)$ & \\
\hline Pathological stage, n (\%) & & & & 0.4 \\
\hline Stage I & $174(73)$ & $164(74)$ & $10(63)$ & \\
\hline Stage II or III & $63(27)$ & $57(26)$ & $6(38)$ & \\
\hline
\end{tabular}

All values are mean $\pm S D$, unless otherwise indicated

$V C$ vital capacity, FEV forced expiratory volume in $1 \mathrm{~s}, F V C$ forced vita capacity, DLCO diffusing capacity of the lung for carbon monoxide, ppoFEV 1 predicted postoperative forced expiratory volume in $1 \mathrm{~s}$, ppoDLCO predicted postoperative diffusing capacity of the lung for carbon monoxide, PA/A ratio pulmonary-artery-to-aorta ratio, $P O C s$ postoperative complications

developed PCCs and 221 did not. Postoperative complications related to surgical procedure (prolonged air leakage in 24 patients and chylothorax in 2 patients) were excluded from the analysis. The mean age of the patients in the PCC group was significantly higher than that of the patients in the non-PCC group (78 \pm 5 years vs $69 \pm$ 9 years, $P=0.0001)$. $P A / A$ ratio was significantly higher in the PCC group than in the non-PCC group $(0.94 \pm$ 0.15 vs $0.81 \pm 0.11, P=0.001)$. Other preoperative patient characteristics, including sex, cardiothoracic ratio, cardiopulmonary comorbidities, Brinkman index, $\mathrm{FEV}_{1}$ as a percentage of the average value in normal individuals $\left(\% \mathrm{FEV}_{1}\right)$, ratio of $\mathrm{FEV}_{1}$ to forced vital capacity $\left(\mathrm{FEV}_{1} /\right.$ FVC), DLCO as a percentage of the average value in normal individuals (\%DLCO), predicted \%ppoFEV $\mathrm{F}_{1}$, and predicted \%ppoDLCO, did not differ significantly between the two groups. Regarding the surgical procedures, bilobectomy was performed in 3 patients, lobectomy in 182 (including 5 bronchovascular reconstructions and 3 resections of involved neighbouring structures), segmentectomy in 42 , and wedge resection in 10 . The proportions of 
patients who underwent lobectomies and other more extensive procedures did not differ significantly between the PCC group and the non-PCC group $(P=1)$. The pathological stage of the disease did not differ significantly between the two groups $(P=0.4)$.

\section{Pulmonary arterial enlargement as a predictor of postoperative cardiopulmonary complications}

Figure 2 shows the area under the ROC curve (AUC), a measure of discriminant or predictive power, to be 0.75 for PA/A ratio. The ratio showed the best combination of sensitivity and specificity for prediction of PCCs at values $>1.0$ ( $44 \%$ sensitivity and $96 \%$ specificity).

\section{Incidence of particular postoperative cardiopulmonary complications and significance of pulmonary arterial enlargement}

Table 2 shows the incidence of particular PCCs. Of the 237 patients in the study sample, 16 developed PCCs for a morbidity rate of $6.8 \%$. Thirteen patients developed one PCC, and 3 developed two PCCs. Atrial fibrillation occurred in 7 patients, pneumonia in 5 , atelectasis in 5 , hypoxemia requiring home oxygen therapy in 3 , and acute heart failure in 1. The overall incidence of PCCs was significantly higher in patients with a PA/A ratio $>1.0$ than in those with a PA/A ratio $\leq 1.0$ ( $35 \%$ vs $4 \%, P=0.0001$ ). The incidences of hypoxemia requiring home oxygen therapy was significantly higher in patients with a PA/A ratio $>1.0$ than in those with a PA/A ratio $\leq 1.0(10 \% \mathrm{vs}$

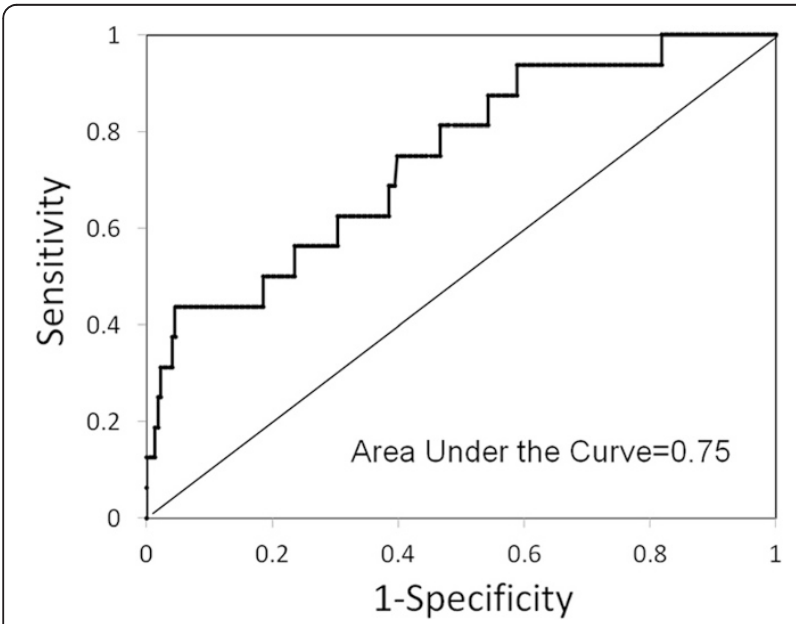

Fig. 2 Receiver operating characteristic curves for PA/A ratio in the prediction of postoperative complications. The area under the receiver operating characteristic curve (AUC), a measure of discriminant or predictive power, was 0.75 for the ratio of the pulmonary artery diameter to the aorta diameter. The ratio showed the best combination of sensitivity and specificity for prediction of postoperative complications at values $>1.0(44 \%$ sensitivity and $96 \%$ specificity)
Table $\mathbf{2}$ Incidence of postoperative cardiopulmonary complications

\begin{tabular}{lllll}
\hline PCC & Total & $\begin{array}{l}\text { PA/A } \\
\text { ratio } \leq 1.0 \\
(n=217)\end{array}$ & $\begin{array}{l}\text { PA/A } \\
\text { ratio }>1.0 \\
(n=20)\end{array}$ & $P$ value \\
\hline Any complication & $16(7)$ & $9(4)$ & $7(35)$ & 0.0001 \\
Atrial fibrillation & $7(3)$ & $5(2)$ & $2(10)$ & 0.11 \\
Pneumonia & $5(2)$ & $3(1)$ & $2(10)$ & 0.06 \\
Atelectasis & $5(2)$ & $3(1)$ & $2(10)$ & 0.06 \\
Hypoxaemia & $3(1)$ & $1(1)$ & $2(10)$ & 0.02 \\
Acute heart failure & $1(0.4)$ & $0(0)$ & $1(0.5)$ & 0.08 \\
30-day mortality & $0(0)$ & $0(0)$ & $(0)$ & 1.0 \\
In-hospital mortality & $1(0.4)$ & $1(0.4)$ & $0(0)$ & 1.0 \\
\hline All values are n (\%) & & & &
\end{tabular}

All values are $\mathrm{n}(\%)$

PCC postoperative cardiopulmonary complication, PA/A ratio

pulmonary-artery-to-aorta ratio

$1 \%, P=0.02)$. The incidences of other particular PCCs did not differ significantly between the two groups. One patient with a PA/A ratio $\leq 1.0$ died from pneumonia on postoperative day 129 for an in-hospital mortality rate of $0.4 \%(1 / 241)$; obviously, the mortality rate did not differ significantly between the two groups $(P=1)$.

\section{Multivariate analysis}

Age $(P=0.0001)$ and PA/A ratio $(P=0.03)$ were significantly different between the PCC and the non-PCC groups on univariate analysis. And cardiopulmonary comorbidities $(P=0.07)$ tended to be more frequent in the PCC group than in the non-PCC group on univariate analysis. These factors were accordingly selected for multivariate logistic regression analysis that identified PA/A ratio (0.1-point increase; odds ratio 2.3, $95 \%$ confidence interval $1.5-3.5 ; P=0.0002)$ and age (1-year increase; odds ratio $1.2,95 \%$ confidence interval $1.1-1.3 ; P=0.003)$ as independent predictors of PCCs (Table 3).

Table 3 Multivariate analysis of patient characteristics possibly contributing to postoperative cardiopulmonary complications

\begin{tabular}{lllll}
\hline Variable & Category & Odds ratio & $95 \% \mathrm{Cl}$ & $P$ value \\
\hline PA/A ratio & Lowest & REF & $1.5-3.5$ & 0.0002 \\
& 0.1-point increase & 2.3 & & \\
Age (years) & Lowest & REF & $1.1-1.3$ & 0.03 \\
& 1-year increase & 1.2 & & \\
Cardiopulmonary & - & REF & $0.7-11.6$ & 0.2 \\
comorbidity & + & 2.5 & & \\
\hline
\end{tabular}

$C /$ confidence interval, $P A / A$ ratio pulmonary-artery-to-aorta ratio, $R E F$ reference parameter 


\section{Preoperative and postoperative pulmonary artery size}

Preoperative and postoperative PA/A ratios are shown in Fig. 3. The postoperative PA/A ratio was significantly higher (median: 0.85 ; 25 th percentile: 0.78 ; 75 th percentile: 0.94$)$ than the preoperative PA/A ratio (median: 0.81; 25th percentile: 0.74 , 75 th percentile: 0.89$)(P<0.0001)$.

\section{Discussion}

In this study, a finding of pulmonary arterial enlargement on computed tomography was associated with the occurrence of PCCs following lung resection for cancer. Of 237 patients who underwent such surgery, 16 developed PCCs for a morbidity rate of $6.8 \%$. The incidence of PCCs was significantly higher in patients with PA/A ratios $>1.0$ than in those with PA/A ratios $\leq 1.0$. Multivariate logistic regression analysis showed that age and PA/A ratio were independent predictors of PCCs.

The present study is the first to show the relationship between pulmonary arterial enlargement on computed tomography and the occurrence of PCCs following lung resection for cancer. It is well known that pulmonary arterial hypertension is associated with various diseases, including left-sided heart disease, chronic obstructive pulmonary disease, interstitial pulmonary disorders, and pulmonary vascular disorders $[15,11,16]$. Moreover, several studies indicate that preoperative pulmonary hypertension may be a predictor of PCCs $[7,6,8]$. As we did not routinely perform Doppler echocardiography, we could not analyze the relationship between PA/A ratio and pulmonary arterial pressure in our patients. However, several previous studies have demonstrated that measurements of pulmonary arterial enlargement based on computed tomography correlate with invasive or echocardiographic measurements of pulmonary arterial pressure [9-11]. Iyer et al. reported that a PA/A ratio $>1.0$ was $73 \%$ sensitive and $84 \%$ specific in identifying patients with mean pulmonary arterial pressure $>25 \mathrm{mmHg}$ [11]. This suggests that the high incidence of PCCs in patients with PA/A ratios $>1.0$ in the present study was caused, at least in part, by pulmonary arterial hypertension. Why is pulmonary hypertension in lung resection patients associated with PCCs? One hypothesis is that pulmonary resection causes the loss of the pulmonary artery bed, which elevates pulmonary arterial pressure and may result in right ventricular dysfunction, particularly in patients who already had pulmonary hypertension preoperatively [17]. Indeed, the current study found PA/A ratios 3 months after surgery to be significantly higher than those before surgery.

Although FEV 1 [18, 2] and DLCO [3, 2] have previously been reported to be strongly predictive of PCCs, they were not significant predictors of PCCs in our study, possibly because we excluded patients with very poor pulmonary function from surgery. In fact, the predicted postoperative pulmonary functions in our patients were very good, with a mean predicted $\%$ ppoFEV $_{1}$ of $87 \% \pm 22 \%$ and a mean predicted \%ppoDLCO of $85 \% \pm 26 \%$.

The calculation of PA/A ratio based on computed tomography is a well-established method for detecting pulmonary hypertension $[12,11,16,9,10]$. Measuring the diameter of main pulmonary artery at the rises of bilateral pulmonary arteries is considered as a reproducible method. Iyer et al. reported the Cohen kappa of 0.82 (95\% CI, 0.68-0.97) for interobserver agreement in the PA/A ratio based on computed tomography. A Cohen's Kappa above 0.8 is regarded as almost perfect agreement. Additionally, the method involves no additional cost and is non-invasive, as all candidates for lung

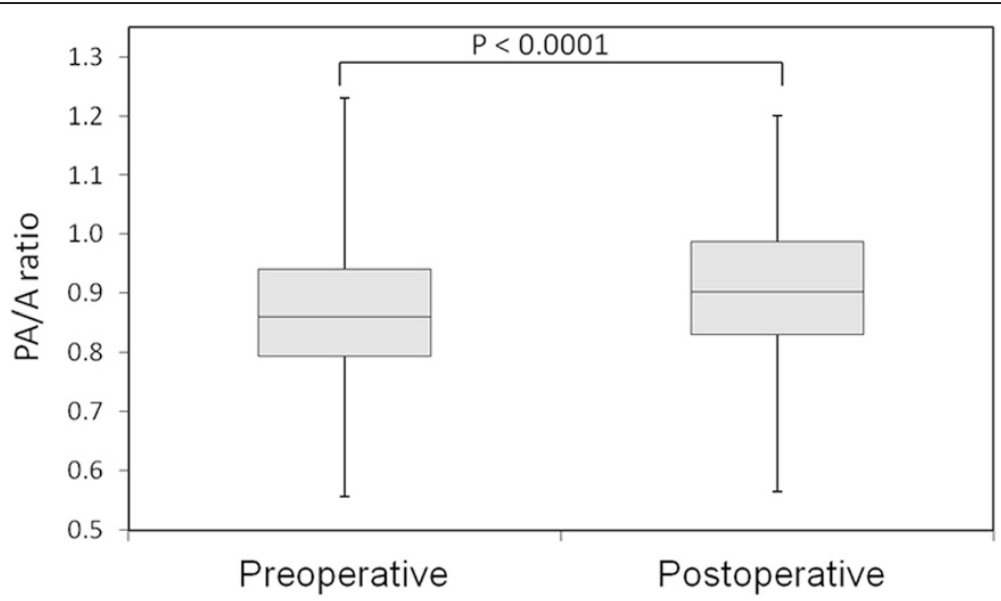

Fig. 3 A box-plot showing preoprerative and postoperative PA/A ratio. A box-plot shows the ratio between the diameters of the pulmonary artery and aorta (PA/A ratio) preoperatively and 3 months after surgery. The postoperative PA/A ratio was significantly higher than the preoperative ratio $(P<0.0001)$ 
resection undergo chest computed tomography preoperatively. Furthermore, the calculation can be completed in approximately $30 \mathrm{~s}$. Given these advantages, $\mathrm{PA} / \mathrm{A}$ ratio is recommended for use in the assessment of candidates for pulmonary resection.

This study had several limitations. Firstly, this study was limited by its retrospective design. Secondly, the number of patients involved in the study was small $(n=241)$. The sample was of similar size to samples in several recent reports from other organizations investigating predictive factors for PCCs following surgery for lung cancer $[14,5,19]$. However the morbidity of PCCs was very low $(6.7 \%$, $\mathrm{n}=16$ ) in the present study, so the number of patients might be inadequate to evaluate the relationship between and PA/A ratio and each particular PCCs. Thirdly, we could not analyze the relationship between PA/A ratio and pulmonary arterial pressure, as we did not routinely perform Doppler echocardiography. Fourthly, cardiopulmonary exercise testing is currently considered the gold standard of risk assessment for lung resection candidates [20]; however, few of the patients in our sample underwent such testing. To further clarify the clinical significance of PA/A ratio in pulmonary resection candidates, large-scale prospective studies investigating its relationships with pulmonary arterial pressure and cardiopulmonary exercise testing are needed.

\section{Conclusions}

A finding of pulmonary arterial enlargement on computed tomography (PA/A ratio $>1.0$ ) is an independent predictor of PCCs following pulmonary resection for lung cancer in this particular study. Further investigation on PA/A ratio in a large-scale prospective study is needed.

\begin{abstract}
Abbreviations
PCC: postoperative cardiopulmonary complication; $\mathrm{FEV}_{1}$ : forced expiratory volume in $1 \mathrm{~s}$; DLCO: diffusing capacity of the lung for carbon monoxide; ppoFEV $V_{1}$ : postoperative forced expiratory volume in $1 \mathrm{~s}$; ppoDLCO: postoperative diffusing capacity of the lung for carbon monoxide; ROC: receiver operating characteristic.
\end{abstract}

\section{Competing interests}

All the authors have no competing interests.

\section{Authors' contributions}

KA designed this study, collected the data, and wrote the manuscript. SM, MT, HS, SO, SM and KK treated the patients, and collected the data. KW supervised the study. All authors read and approved the final manuscript.

\section{Acknowledgements}

We thank the staff of the physiology laboratory of Sagamihara Kyodo Hospital for performing the pulmonary function tests.

\section{Funding}

This work was not supported by any external funding.

\section{Author details}

'Division of Thoracic Surgery, Department of Surgery, School of Medicine, Keio University, 35 Shinanomachi, Shinjuku-ku, Tokyo, Japan. ${ }^{2}$ Department of General Thoracic Surgery, Sagamihara Kyodo Hospital, Kanagawa, Japan.
Received: 7 February 2015 Accepted: 24 August 2015

Published online: 09 September 2015

\section{References}

1. Boffa DJ, Allen MS, Grab JD, Gaissert HA, Harpole DH, Wright CD. Data from The Society of Thoracic Surgeons General Thoracic Surgery database: the surgical management of primary lung tumors. J Thorac Cardiovasc Surg. 2008;135(2):247-54. doi:10.1016/j.jtcvs.2007.07.060.

2. Salati M, Refai M, Pompili C, Xiume F, Sabbatini A, Brunelli A. Major morbidity after lung resection: a comparison between the European Society of Thoracic Surgeons Database system and the Thoracic Morbidity and Mortality system. J Thorac Dis. 2013;5(3):217-22. doi:10.3978/j.issn.2072-1439.2013.05.03.

3. Ferguson MK, Vigneswaran WT. Diffusing capacity predicts morbidity after lung resection in patients without obstructive lung disease. Ann Thorac Surg. 2008;85(4):1158-64. doi:10.1016/j.athoracsur.2007.12.071. discussion 64-5.

4. Brunelli A, Belardinelli R, Pompili C, Xiume F, Refai M, Salati M, et al. Minute ventilation-to-carbon dioxide output (VENCO2) slope is the strongest predictor of respiratory complications and death after pulmonary resection. Ann Thorac Surg. 2012;93(6):1802-6. doi:10.1016/j.athoracsur.2012.03.022.

5. Bobbio A, Chetta A, Internullo E, Ampollini L, Carbognani P, Bettati S, et al. Exercise capacity assessment in patients undergoing lung resection. Eur J Cardiothorac Surg. 2009;35(3):419-22. doi:10.1016/j.ejcts.2008.11.004.

6. Rams JJ, Harrison RW, Fry WA, Moulder PV, Adams WE. Operative pulmonary artery pressure measurements as a guide to postoperative management and prognosis following pneumonectomy. Dis Chest. 1962;41:85-90.

7. Fee HJ, Holmes EC, Gewirtz HS, Ramming KP, Alexander JM. Role of pulmonary vascular resistance measurements in preoperative evaluation of candidates for pulmonary resection. J Thorac Cardiovasc Surg. 1978;75(4):519-24.

8. Pierce RJ, Sharpe K, Johns J, Thompson B. Pulmonary artery pressure and blood flow as predictors of outcome from lung cancer resection. Respirology. 2005;10(5):620-8. doi:10.1111/j.1440-1843.2005.00759.x.

9. Devaraj A, Wells AU, Meister MG, Corte TJ, Wort SJ, Hansell DM. Detection of pulmonary hypertension with multidetector $\subset$ and echocardiography alone and in combination. Radiology. 2010;254(2):609-16. doi:10.1148/radiol.09090548.

10. Perez-Enguix D, Morales P, Tomas JM, Vera F, Lloret RM. Computed tomographic screening of pulmonary arterial hypertension in candidates for lung transplantation. Transplant Proc. 2007;39(7):2405-8. doi:10.1016/j.transproceed.2007.07.055.

11. Iyer AS, Wells JM, Vishin S, Bhatt SP, Wille KM, Dransfield MT. CT scanmeasured pulmonary artery to aorta ratio and echocardiography for detecting pulmonary hypertension in severe COPD. Chest. 2014;145(4):824-32. doi:10.1378/chest.13-1422

12. Wells JM, Washko GR, Han MK, Abbas N, Nath H, Mamary AJ, et al. Pulmonary arterial enlargement and acute exacerbations of COPD. N Engl J Med. 2012;367(10):913-21. doi:10.1056/NEJMoa1203830.

13. Brunelli A, Refai M, Xiume F, Salati M, Marasco R, Sciarra V, et al. Oxygen desaturation during maximal stair-climbing test and postoperative complications after major lung resections. Eur J Cardiothorac Surg. 2008;33(1):77-82. doi:10.1016/j.ejcts.2007.09.028.

14. Ambrozin AR, Cataneo DC, Arruda KA, Cataneo AJ. Time in the stairclimbing test as a predictor of thoracotomy postoperative complications. J Thorac Cardiovasc Surg. 2013;145(4):1093-7. doi:10.1016/j.jtcvs.2012.09.001.

15. Rich S, Rabinovitch M. Diagnosis and treatment of secondary (noncategory 1) pulmonary hypertension. Circulation. 2008;118(21):2190-9. doi:10.1161/CIRCULATIONAHA.107.723007.

16. Devaraj A, Wells AU, Meister MG, Loebinger MR, Wilson R, Hansell DM. Pulmonary hypertension in patients with bronchiectasis: prognostic significance of $C T$ signs. AJR Am J Roentgenol. 2011;196(6):1300-4. doi:10.2214/AJR.10.5221.

17. Okada M, Ota T, Matsuda H, Okada K, Ishii N. Right ventricular dysfunction after major pulmonary resection. J Thorac Cardiovasc Surg. 1994;108(3):503-11.

18. Miller Jr Jl. Physiologic evaluation of pulmonary function in the candidate for lung resection. J Thorac Cardiovasc Surg. 1993;105(2):347-51. discussion 51-2.

19. Brunelli A, Charloux A, Bolliger CT, Rocco G, Sculier JP, Varela G, et al. The European Respiratory Society and European Society of Thoracic Surgeons clinical guidelines for evaluating fitness for radical treatment (surgery and chemoradiotherapy) in patients with lung cancer. Eur I Cardiothorac Surg. 2009;36(1):181-4. doi:10.1016/j.ejcts.2009.04.022.

20. Brunelli A, Charloux A, Bolliger CT, Rocco G, Sculier JP, Varela G, et al. ERS/ESTS clinical guidelines on fitness for radical therapy in lung cancer patients (surgery and chemo-radiotherapy). Europ Respir J. 2009;34(1):17-41. doi:10.1183/09031936.00184308. 